\title{
SCHUBERT PATCHES DEGENERATE TO SUBWORD COMPLEXES
}

\author{
ALLEN KNUTSON \\ Dedicated to Bert Kostant on the occasion of his 80th birthday
}

\begin{abstract}
We study the intersections of general Schubert varieties $X_{w}$ with permuted big cells, and give an inductive degeneration of each such "Schubert patch" to a StanleyReisner scheme. Similar results had been known for Schubert patches in various types of Grassmannians. We maintain reducedness using the results of [Knutson 2007] on automatically reduced degenerations, or through more standard cohomology-vanishing arguments.

The underlying simplicial complex of the Stanley-Reisner scheme is a subword complex, as introduced for slightly different purposes in [Knutson-Miller 2004], and is homeomorphic to a ball. This gives a new proof of the Andersen-Jantzen-Soergel/Billey and Graham/Willems formulae for restrictions of equivariant Schubert classes to fixed points.
\end{abstract}

\section{CONTENTS}

0.1. Cohomology: Schubert classes in equivariant K-theory 1

0.2. Geometry: Schubert patches 2

0.3. Combinatorics: subword complexes 4]

0.4. From \#2 to \#3: Schubert patches degenerate to subword complexes 8

0.5. Acknowledgements 10

References $\quad 10$

Fix a pinning $\left(G, T, W, N_{ \pm}, B_{ \pm}=T_{ \pm}\right)$of a complex reductive Lie group $G$. Then there are correspondences between the Weyl group $W:=N(T) / T$, the $B_{-}$-orbits on the flag manifold $\mathrm{G} / \mathrm{B}_{+}$, and the set of T-fixed points $\left(\mathrm{G} / \mathrm{B}_{+}\right)^{\mathrm{T}}$, namely $w \mapsto \mathrm{B}_{-} w \mathrm{~B}_{+} / \mathrm{B}_{+}, w \mathrm{~B}_{+} / \mathrm{B}_{+}$. Let $\mathrm{X}_{w}:=\overline{\mathrm{B}_{-} w \mathrm{~B}_{+}} / \mathrm{B}_{+} \subseteq \mathrm{G} / \mathrm{B}_{+}$be the Schubert variety associated to $w \in W$.

0.1. Cohomology: Schubert classes in equivariant K-theory. The T-invariant cycles $\left\{X_{w}\right\}$ define bases in many (co)homology theories of $G / B$, in particular T-equivariant ones. We will focus our attention on equivariant $\mathrm{K}$-theory $\mathrm{K}_{\mathrm{T}}(\mathrm{G} / \mathrm{B})$, an algebra over the (Laurent polynomial) ring $\mathrm{K}_{\mathrm{T}}(\mathrm{pt})$ of virtual characters of the torus $\mathrm{T}$. However, the results we mention have (and imply) analogues for equivariant cohomology.

The structure sheaf of $X_{w}$ defines an element $S_{w} \in K_{T}(G / B)$. These classes $S_{w}$ were first calculated by Demazure (implicitly, in his formula [De74] for the characters of Demazure modules), though his calculation had a gap (later filled) which we will come to in a moment.

Date: Hallowe'en 2007.

Supported by an NSF grant. 
In the paper [KosKu90] by Bertram Kostant and Shrawan Kumar, they suggest that one describe $S_{w}$ using the restriction map (and $\mathrm{K}_{\mathrm{T}}(\mathrm{pt})$-algebra homomorphism)

$$
\mathrm{K}_{\mathrm{T}}(\mathrm{G} / \mathrm{B}) \hookrightarrow \mathrm{K}_{\mathrm{T}}\left((\mathrm{G} / \mathrm{B})^{\mathrm{T}}\right)=\bigoplus_{w \in W} \mathrm{~K}_{\mathrm{T}}(\mathrm{pt})
$$

The key point is that this map is injective, so no information is lost by localizing to fixed points. To match notation with [KosKu90] we think of the weight lattice $T^{*}$ additively, and denote the class of the 1-dimensional representation with weight $\lambda \in T^{*}$ by $e^{\lambda} \in K_{T}(p t)$.

Let $S_{\left.w\right|_{v}} \in K_{T}(p t)$ denote the restriction of the class $S_{w}$ to the T-fixed point $v$. Then $\left.S_{w}\right|_{v}$ can be calculated inductively in $v$ :

Theorem 1. Let $v, w$ be elements of $W$. If $\left.S_{w}\right|_{v} \neq 0$, then $v \geq w$ in the Bruhat order. Assume this hereafter.

If $v=1$, then $w=1$ and $\left.S_{w}\right|_{v}=1$. Otherwise, there exists a simple root $\alpha$ such that $v r_{\alpha}<v$ in the Bruhat order (equivalently, $v \cdot \alpha$ is a negative root). Then $\left.S_{w}\right|_{v}$ can be computed from $\left.S_{w}\right|_{v r_{\alpha}}$ and $\left.\mathrm{S}_{w \mathrm{r}_{\alpha}}\right|_{v \mathrm{r}_{\alpha}}$ :

(1) If $w r_{\alpha}>w$, then $\left.S_{w}\right|_{v}=\left.S_{w}\right|_{v r_{\alpha}}$.

(2) If $w r_{\alpha}<w$ but $w \not \leq v r_{\alpha}$, then $\left.S_{w}\right|_{v}=\left.\left(1-e^{v \cdot \alpha}\right) S_{w r_{\alpha}}\right|_{v r_{\alpha}} \cdot$

(3) If $w r_{\alpha}<w \leq v r_{\alpha}$, then $\left.S_{w}\right|_{v}=\left.S_{w}\right|_{v r_{\alpha}}+\left.\left(1-e^{v \cdot \alpha}\right) S_{w r_{\alpha}}\right|_{v r_{\alpha}}-\left.\left(1-e^{v \cdot \alpha}\right) S_{w}\right|_{v r_{\alpha}}$.

(In fact (3) includes (2).)

This is essentially in [KosKu90] stated using Demazure operators, and also follows trivially from the Graham/Willems formulae for $\left\{\left.S_{w}\right|_{v}\right\}$ [Gr02, Wi06]. One can give a reasonably straightforward direct proof (in the finite-dimensional case) if one computes with the Bott-Samelson-Demazure-Hansen resolutions of Schubert varieties. However, such a proof depends on the fact that the map from the Bott-Samelson manifold to the Schubert variety takes the fundamental K-class to the fundamental K-class, which can be proven by showing that the higher direct images vanish of the pushforward of the structure sheaf (i.e. that Schubert varieties have rational singularities). This was exactly the gap in Demazure's argument; an account of this (and how the gap was eventually filled using characteristic $p$ methods) can be found in [Ja87, chapter 14].

In theorem 2 we will give a geometric interpretation (and new proof) of theorem 1 , based on the degenerations of [Kn] applied to "Schubert patches". In theorem 3 we will give a new combinatorial interpretation of theorem 1, based on vertex decompositions of the "subword complexes" of [KnM05, KnM04]. In our principal new theorem, theorem 4. we will relate the geometry and the combinatorics directly by giving a degeneration of Schubert patches to Stanley-Reisner schemes of subword complexes.

0.2. Geometry: Schubert patches. One thing we do in this paper is to replace the use of resolutions with degenerations, and replace the cohomology-vanishing arguments with lemmas from [ $[\mathrm{Kn}]$ about automatically reduced degenerations. These lemmas are in turn based on the theory of branchvarieties [AK], though we will not need to inspect those underpinnings in the present paper.

We define $\left.X_{w}\right|_{v}:=X_{w} \cap\left(v N_{-} B_{+} / B_{+}\right)$as the intersection of $X_{w}$ with the permuted big cell $v N_{-} B_{+} / B_{+}$, and call it a Schubert patch on $X_{w}$, as the $\left\{\left.X_{w}\right|_{v}, v \geq w\right\}$ form an affine open cover of the Schubert variety $X_{w}$. Each Schubert patch carries an action of $T$, and the 
T-equivariant transverse pullback diagram

$$
\begin{array}{cccc}
\left.\mathrm{X}_{w}\right|_{v} & \hookrightarrow & \mathrm{X}_{w} \\
\downarrow & & & \downarrow \\
v \mathrm{~B}_{+} / \mathrm{B}_{+} \hookrightarrow & v \mathrm{~N}_{-} \mathrm{B}_{+} / \mathrm{B}_{+} & \hookrightarrow & \mathrm{G} / \mathrm{B}_{+}
\end{array}
$$

shows that we can compute the restriction $\left.S_{w}\right|_{v}$ as the class $\left[\left.X_{w}\right|_{v}\right] \in K_{T}\left(v N_{-} B_{+} / B_{+}\right) \cong K_{T}(p t)$. This is convenient for a number of purposes, one being that the permuted big cell $v \mathrm{~N}_{-} \mathrm{B}_{+} / \mathrm{B}_{+}$ is a vector space whose T-weights all live in the interior of a half-space of $\mathrm{T}^{*}$. As such, $\mathrm{X}_{w} \mathrm{l}_{v}$ has a multigraded Hilbert series, and the $\mathrm{K}_{\mathrm{T}}$-class $\left.S_{w}\right|_{v}$ is essentially this series times $v \cdot$ (the Weyl denominator); see [MS05, chapter 8.2]. This is the viewpoint of [GR06, KodR03, KrL04, RU\#1, RU\#2].

The degenerations we use are of a very specific type, which we christened geometric vertex decompositions in [KnMY]. As explained in [Kn, section 4.1], the permuted big cell $\nu \mathrm{N}_{-} \mathrm{B}_{+} / \mathrm{B}_{+} \cong \mathrm{N}_{-}$factors T-equivariantly as a product of a line $\mathrm{L}$ with weight $-v \cdot \alpha$ and a complementary hyperplane $H$. Let the multiplicative group $\mathbb{G}_{m}$ act on the permuted big cell $\mathrm{H} \times \mathrm{L}$ by scaling $\mathrm{L}$, and define

$$
X^{\prime}:=\lim _{t \rightarrow 0} t \cdot X_{w \mid v}
$$

as the scheme-theoretic limit. Automatically, $X^{\prime}$ has the same multigraded Hilbert series and (equivalently) defines the same $K_{T}$-class as $\left.X_{w}\right|_{v}$.

We can now state our degeneration-based analogue of theorem 11. In it, we use the notation $\mathbb{A}_{\lambda}^{1}$ to denote the 1-dimensional T-representation with weight $\lambda$, and all isomorphisms stated are T-equivariant.

Theorem 2. Let $v, w$ be elements of $W$. If $\left.X_{w}\right|_{v} \neq \emptyset$, then $v \geq w$ in the Bruhat order. Assume this hereafter.

If $v=1$, then $w=1$ and $\left.\mathrm{X}_{w}\right|_{v}=\mathrm{N}_{-} \mathrm{B}_{+} / \mathrm{B}_{+}$. Otherwise, there exists a simple root $\alpha$ such that $v \mathrm{r}_{\alpha}<v$ in the Bruhat order. Let $\mathrm{X}^{\prime}$ be the degeneration of $\left.\mathrm{X}_{w}\right|_{v}$ described above.

(1) If $w r_{\alpha}>w$, then $X^{\prime}=\left.X_{w}\right|_{v}$ (the limiting process is trivial), and

$$
\left.X_{w}\right|_{v} \cong \Pi \times \mathbb{A}_{-v \cdot \alpha}^{1},\left.\quad X_{w}\right|_{v r_{\alpha}} \cong \Pi \times \mathbb{A}_{v \cdot \alpha}^{1}
$$

for the same $\Pi$.

(2) If $w r_{\alpha}<w$ but $w \not \leq v r_{\alpha}$, then $X^{\prime}=\left.X_{w}\right|_{v}$ (again, the limiting process is trivial), and

$$
\left.\left.X_{w r_{\alpha}}\right|_{v r_{\alpha}} \cong X_{w}\right|_{v} \times \mathbb{A}_{v \cdot \alpha}^{1} \text {. }
$$

(3) If $w r_{\alpha}<w \leq v r_{\alpha}$, then $X^{\prime}$ is reduced, and has two components:

$$
X^{\prime}=(\Pi \times\{0\}) \cup_{\Lambda \times\{0\}}\left(\Lambda \times \mathbb{A}_{-v \cdot \alpha}^{1}\right)
$$

where $\Pi \times\left.\mathbb{A}_{v \cdot \alpha}^{1} \cong X_{w r_{\alpha}}\right|_{v r_{\alpha}}$ and $\Lambda \times\left.\mathbb{A}_{v \cdot \alpha}^{1} \cong X_{w}\right|_{v r_{\alpha}}$.

Proof. Parts (1) and (2) are proposition 6 of $[\mathrm{Kn}]$ and are surely well-known to the experts. Part (3), which is much deeper, is proposition 7 of $[\mathrm{Kn}]$. (This is why we separated cases (2) and (3) in theorem 1)

By manipulating Hilbert series, it is easy to recover each part of theorem 1 from the corresponding part of theorem 2. And indeed, while the set-theoretic description of $X^{\prime}$ is reasonably straightforward [KnMY, theorem $2.2 \mathrm{~b}]$, its reducedness is essentially equivalent to the Kostant-Kumar recursion. But where part (3) of theorem 1 was proven using 
the vanishing of higher cohomology of the Bott-Samelson-Demazure-Hansen resolution, part (3) of theorem [2] is based on [Kn, proposition 7], which shows that the (by definition reduced) "limit branchvariety" coincides with the limit subscheme.

Having a degeneration implies more than merely an equality of $\mathrm{K}_{\mathrm{T}}$-classes: in [ $\mathrm{Kn}$ ] we use these results to give a new proof that Schubert varieties are normal and CohenMacaulay.

In the remainder of the paper it will be convenient to work not directly with Schubert patches, but the more economical Kazhdan-Lusztig varieties $X_{w o}^{v}:=X_{w} \cap X_{\circ}^{v}$, where $X_{\circ}^{v}$ denotes the opposite Schubert cell $\mathrm{N}_{+} v \mathrm{~B}_{+} / \mathrm{B}_{+}$. These are obtained from Schubert patches by factoring out a largely irrelevant vector space:

Lemma 1. [KaL79, Lemma A.4] Let $w, v \in W$. Then there is a T-equivariant factorization

$$
\left.X_{w}\right|_{v} \cong X_{w_{0}}^{v} \times X_{v}^{\circ}
$$

where $X_{v}^{\circ}:=\mathrm{N}_{-} v \mathrm{~B}_{+} / \mathrm{B}_{+}$is just a T-vector space, with weights $\{\beta<0: v \cdot \beta<0\}$. In particular the dimension of the Kazhdan-Lusztig variety is $\operatorname{dim} X_{w o}^{v}=\ell(v)-\ell(w)$.

Each result about Schubert patches has an equivalent, though often simpler, version for Kazhdan-Lusztig varieties. The only possibly subtle one is the restriction of the geometric vertex decomposition, which is a family of subschemes of $\left.\left.X_{1}\right|_{v} \cup X_{1}\right|_{v r_{\alpha}}$; for this one intersects each fiber of the family with $X^{v}$, obtaining a family of subschemes of $X_{\circ}^{v} \cup X_{\circ}^{v r_{\alpha}}$.

Theorem 2]. Let $v, w$ be elements of W. If $X_{w o}^{v} \neq \emptyset$, then $v \geq w$ in the Bruhat order. Assume this hereafter.

If $v=1$, then $w=1$ and $X_{w o}^{v}=B_{+} / B_{+}$. Otherwise, there exists a simple root $\alpha$ such that $v \mathrm{r}_{\alpha}<v$ in the Bruhat order.

(1) If $w r_{\alpha}>w$, then $X_{w o}^{v} \cong X_{w o}^{v r_{\alpha}} \times \mathbb{A}_{-v \cdot \alpha}^{1}$.

(2) If $w r_{\alpha}<w$ but $w \leq \leq v r_{\alpha}$, then $X_{w o}^{v} \cong X_{w r_{\alpha}}^{v r_{\alpha}}$.

(3) If $w r_{\alpha}<w \leq v r_{\alpha}$, then $X^{\prime}$ is reduced, and has two components:

$$
\begin{aligned}
& X^{\prime}=(\Pi \times\{0\}) \cup_{\Lambda \times\{0\}}\left(\Lambda \times \mathbb{A}_{-v \cdot \alpha}^{1}\right) \\
& \text { where } \Pi \cong X_{w r_{\alpha} \circ}^{v r_{\alpha}} \text { and } \Lambda \cong X_{w o}^{v r_{\alpha}} \text {. }
\end{aligned}
$$

0.3. Combinatorics: subword complexes. When attempting to unwind theorem 1 to a direct formula for $\left.S_{w}\right|_{v}$, as in Kumar's appendix to [Bi99], one is led naturally to the definition of a subword complex [KnM04] (though our motivation at the time was slightly different).

Let $\mathrm{Q}=\left(\alpha_{1}, \alpha_{2}, \ldots, \alpha_{k}\right)$ be a sequence of simple roots such that $v=\prod_{i=1}^{k} r_{\alpha_{i}}$, and $k$ is minimized. Then $Q$ is called a reduced word for $v$, and $k$ its length, usually denoted $\ell(v)$. (Warning: because we use the Kostant-Kumar recurrence based on $v r_{\alpha}$ and not one based on $r_{\alpha} \nu$, the first root used in applying the recurrence is $\alpha_{k}$, not $\alpha_{1}$.)

In [KnM05, KnM04] we defined the subword complex $\Delta(\mathrm{Q}, w)$ associated to a reduced word $Q$ and a Weyl group element $w$ as the simplicial complex whose vertex set is $Q$ (or really, 1...k) with $F \subseteq Q$ a facet (maximal face) iff the complement $Q \backslash F$ is a reduced word for $w$.

Even when $Q \backslash F$ is not a reduced word, we can define its Demazure product by multiplying the reflections in order, omitting along the way any one that brings us lower in the Bruhat order. (Equivalently, one may take the product of any maximal reduced subword.) 
Proposition 1. [KnM04] The subword complex $\Delta(\mathrm{Q}, w)$ is homeomorphic to a ball or sphere; in particular every ridge (codimension 1 face) is contained in one or two facets.

For any face $\mathrm{F} \in \Delta(\mathrm{Q} ; w)$, the Demazure product of $\mathrm{Q} \backslash \mathrm{F}$ is $\geq w$ in the Bruhat order, with equality iff $\mathrm{F}$ is an interior face (i.e. if $\mathrm{F}$ is contained in no ridge contained in only one facet).

To any simplicial complex $\Delta$ on vertex set $Q$, and a field $\mathbb{F}$, one may associate the (affine) Stanley-Reisner scheme $\mathrm{SR}(\Delta) \subseteq \mathbb{F}^{\mathrm{Q}}$, the union of the corresponding coordinate planes:

$$
\operatorname{SR}(\Delta):=\bigcup_{S \in \Delta} \mathbb{F}^{\mathrm{S}}
$$

These schemes are invariant under the action of the torus $\left(\mathbb{G}_{m}\right)^{\mathrm{Q}}$ that dilates the coordinates independently. (Indeed, they are characterized by this invariance plus their reducedness; note too that $\Delta$ can be reconstructed from $\operatorname{SR}(\Delta)$.) As such $\operatorname{SR}(\Delta)$ has an associated multigraded Hilbert series in the variables $\left(q_{1}, \ldots, q_{|Q|}\right)$ :

$$
\left.h_{S R(\Delta)}=\sum \text { (those monomials whose variables form a face in } \Delta\right)=\sum_{F \in \Delta} \prod_{q_{j} \in F} \frac{q_{j}}{1-q_{j}} .
$$

Equivalently, one can compute the class $k_{\Delta}:=[\operatorname{SR}(\Delta)] \in \mathrm{K}_{\left(\mathbb{G}_{\mathrm{m}}\right) \mathrm{Q}}\left(\mathbb{F}^{\mathrm{Q}}\right)$, by

$$
k_{\Delta}=\left(\prod_{q_{j}}\left(1-q_{j}\right)\right) h_{S R(\Delta)}=\sum_{F \in \Delta} \prod_{q_{j} \in F} q_{j} \prod_{q_{j} \notin F}\left(1-q_{j}\right) .
$$

One can give an alternate formula for the Hilbert series $h_{\mathrm{SR}(\Delta)}$ in which the summands are of the form $\prod_{q_{j} \in F} 1 /\left(1-q_{j}\right)$ rather than $\prod_{q_{j} \in F} q_{j} /\left(1-q_{j}\right)$, corresponding to writing $\Delta$ as a union of closed faces rather than open faces. The resulting inclusion-exclusion of the faces is particularly simple in the case of $\Delta$ a ball or sphere, and becomes an alternating sum over the interior faces:

Corollary 1. [KnM04, Lemma 4.2]

$$
\mathrm{h}_{\mathrm{SR}(\Delta(\mathrm{Q}, w))}=\sum_{\substack{\mathrm{F} \subseteq \mathrm{Q} \\ \Pi(\mathrm{Q} \backslash \mathrm{F})=w}}(-1)^{|\mathrm{Q} \backslash \mathrm{F}|} \prod_{\mathrm{q}_{\mathrm{j}} \in \mathrm{F}} \frac{1}{1-\mathrm{q}_{j}}
$$

where $\prod(\mathrm{Q} \backslash \mathrm{F})$ means the Demazure product of the subword.

We comment that if one sums over all faces of $\Delta(\mathrm{Q}, w)$, not just the interior ones, one obtains the $\mathrm{K}_{\mathrm{T}}$-class of the ideal sheaf of the "boundary" of the Schubert variety. These ideal sheaves give another $\mathrm{K}_{\mathrm{T}}(\mathrm{pt})$-basis of $\mathrm{K}_{\mathrm{T}}(\mathrm{G} / \mathrm{B})$ which (upon twisting by the long element in $W$ ) are the dual basis under the Poincare pairing on $\mathrm{K}_{\mathrm{T}}(\mathrm{G} / \mathrm{B})$, and this formula for them appears in [Gr02, section 3].

To give a simplicial-complex analogue of theorem 1, we will need an analogue of the decomposition that appears in its part (3). The deletion of a vertex $p$ from a simplicial complex $\Delta$ is the subcomplex $\operatorname{del}_{\mathrm{p}} \Delta:=\{\mathrm{F} \in \Delta: \mathrm{F} \not \supset \mathrm{p}\}$, and the star of the vertex $p$ is the subcomplex star $\operatorname{st}_{\mathrm{p}}:=\{\mathrm{F} \in \Delta: \mathrm{F} \cup\{\mathrm{p}\} \in \Delta\}$. So $\mathrm{p}$ is a cone point of its star, and deleting it we get the link link $k_{p} \Delta$ of $p$. The decomposition

$$
\Delta=\operatorname{del}_{p} \Delta \cup_{\operatorname{link}_{\mathrm{p}} \Delta} \operatorname{star}_{\mathrm{p}} \Delta
$$


is a vertex decomposition, as used in [BP79]. At the risk of getting ahead of ourselves, we mention that this should be seen as analogous to part (3) of theorem 2 (hence the term "geometric vertex decomposition"), with $\operatorname{del}_{p} \Delta$ and $\operatorname{link}_{p} \Delta$ playing the roles of $\Pi$ and $\Lambda$.

Theorem 3. [KnM04] Let $v, w$ be elements of $W$. Let $\mathrm{Q}$ be a reduced word for $v$. If $\Delta(\mathrm{Q}, w) \neq \emptyset$, then $v \geq w$ in the Bruhat order. Assume this hereafter.

If $v=1$, then $w=1, Q=()$ and $\Delta(\mathrm{Q}, w)=\{\emptyset\}$. Otherwise, let $\alpha$ be the last simple root listed in $\mathrm{Q}$, and let $\mathrm{Q}^{\prime}$ be $\mathrm{Q}$ with this root dropped. (Hence $v \mathrm{r}_{\alpha}<v$.)

(1) If $w r_{\alpha}>w$, then $\ell(v)$ is a cone point of $\Delta(Q, w)$, and

$$
\operatorname{link}_{\ell(v)} \Delta(\mathrm{Q}, w)=\operatorname{del}_{\ell(v)} \Delta(\mathrm{Q}, w)=\Delta\left(\mathrm{Q}^{\prime}, w\right) .
$$

(2) If $w r_{\alpha}<w$ but $w \not \leq v r_{\alpha}$, then no face of $\Delta(Q, w)$ uses $\ell(v)$, and

$$
\Delta(\mathrm{Q}, w)=\operatorname{del}_{\ell(v)} \Delta(\mathrm{Q}, w)=\Delta\left(\mathrm{Q}^{\prime}, w \mathrm{r}_{\alpha}\right) .
$$

(3) If $w r_{\alpha}<w \leq v r_{\alpha}$, then the vertex decomposition at the vertex $\ell(v)$ is into

$$
\operatorname{link}_{\ell(v)} \Delta(\mathrm{Q}, w)=\Delta\left(\mathrm{Q}^{\prime}, w\right), \quad \operatorname{del}_{\ell(v)} \Delta(\mathrm{Q}, w)=\Delta\left(\mathrm{Q}^{\prime}, w \mathrm{r}_{\alpha}\right) .
$$

Proof. The proofs are all largely tautologies based on the Bruhat order, taking care not to be confused by the complementation involved in the definition of subword complex.

(1) If $w r_{\alpha}>w$, then the last letter in a reduced word for $w$ cannot be $r_{\alpha}$. Complementing, $\ell(v)$ must lie in every facet of $\Delta(\mathrm{Q}, w)$. Then any subword of $\mathrm{Q}$ using the first $\ell(v)$ letters but avoiding $\ell(v)$ is equivalently a subword of $Q$ using the first $\ell(v)-1$ letters, i.e. of $\mathrm{Q}^{\prime}$.

(2) If $w \not \leq v r_{\alpha}$, then no subword of the first $\ell(v)-1$ letters in Q has product $w$. Hence any subword of the first $\ell(v)$ letters with product $w$ must use the $\ell(v)$ th letter. Removing that letter, we get the product $w r_{\alpha}$.

(3) As the answer suggests, this is essentially a combination of the previous two arguments.

(As in theorem 1, (2) is really a subproblem of (3). It is only in theorem 2 that it is in any way natural to separate them.)

We are ready to connect theorems 1 and 3, the equivariant $\mathrm{K}$-classes computed in theorem 1 can be computed from the Hilbert series associated to subword complexes.

Corollary 2. Fix $\mathrm{Q}, v, w$ as above, and let

$$
\beta_{j}:=\left(\prod_{k=1}^{j-1} r_{\alpha_{k}}\right) \cdot \alpha_{j}, \quad j=1, \ldots, \ell(v) .
$$

Then the specialization

$$
\mathrm{h}_{\mathrm{Q}, w}:=\mathrm{h}_{\mathrm{SR}(\Delta(\mathrm{Q}, w))} \text { with } \mathrm{q}_{\mathrm{i}} \mapsto \mathrm{e}^{\beta_{\mathrm{i}}}
$$

of the Hilbert series of the Stanley-Reisner scheme of the subword complex is the T-equivariant Hilbert series of the Kazhdan-Lusztig variety $\mathrm{S}_{w_{0}}^{v}$. Equivalently,

$$
\left.S_{w}\right|_{v}=\left(\prod_{\substack{\beta>0 \\ v \cdot \beta>0}}\left(1-e^{-v \cdot \beta}\right)\right) h_{\mathrm{Q}, w}
$$

where the product is over those positive roots $\beta$ of $\mathrm{G}$ that stay positive when twisted by $v$. 
Proof. Assume $v \geq w$, for otherwise both sides are zero. Then if $v=1$, both sides are 1 . Otherwise $\ell(v) \geq 1$ and as before we let $\mathrm{Q}^{\prime}$ be $\mathrm{Q}$ minus its last letter.

Theorem 3 implies corresponding results for the Hilbert series and K-polynomials:

(1) If $w r_{\alpha}>w$, then

$$
\mathrm{h}_{\Delta(\mathrm{Q}, w)}=\mathrm{h}_{\Delta\left(\mathrm{Q}^{\prime}, w\right)} /\left(1-\mathrm{q}_{\ell(v)}\right), \quad \mathrm{k}_{\mathrm{Q}, w}=\mathrm{k}_{\mathrm{Q}^{\prime}, w} .
$$

(2) If $w r_{\alpha}<w$ but $w \not \leq v r_{\alpha}$, then

$$
\mathrm{h}_{\Delta(\mathrm{Q}, w)}=\mathrm{h}_{\Delta\left(\mathrm{Q}^{\prime}, w r_{\alpha}\right)}, \quad \mathrm{k}_{\mathrm{Q}, w}=\left(1-\mathrm{q}_{\ell(v)}\right) \mathrm{k}_{\mathrm{Q}^{\prime}, w r_{\alpha}} .
$$

(3) If $w r_{\alpha}<w \leq v r_{\alpha}$, then

$$
\begin{aligned}
& \mathrm{h}_{\Delta(\mathrm{Q}, w)}=\mathrm{h}_{\Delta\left(\mathrm{Q}^{\prime}, w\right)} /\left(1-\mathrm{q}_{\ell(v)}\right)+\mathrm{h}_{\Delta\left(\mathrm{Q}^{\prime}, w r_{\alpha}\right)}-\mathrm{h}_{\Delta\left(\mathrm{Q}^{\prime}, w\right)} \\
& \mathrm{k}_{\mathrm{Q}, w}=\mathrm{k}_{\mathrm{Q}^{\prime}, w}+\left(1-\mathrm{q}_{\ell(v)}\right) \mathrm{k}_{\mathrm{Q}^{\prime}, w r_{\alpha}}-\left(1-\mathrm{q}_{\ell(v)}\right) \mathrm{k}_{\mathrm{Q}^{\prime}, w} .
\end{aligned}
$$

Under the specialization $q_{i} \mapsto e^{\beta_{i}}$ of $k_{Q, w}$, we recover the equations from theorem 1 , So under this specialization, $\left.S_{w}\right|_{v}=k_{Q, w}$, and $k_{Q, w}=\left(\prod_{\substack{\beta>\beta>0 \\ v>>>0}}\left(1-e^{-v \cdot \beta}\right)\right) h_{Q, w}$. This establishes the second claim.

For the first, we use the transversality of the pullback diagram

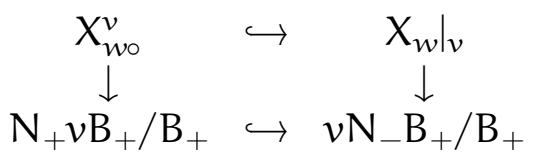

and the relation of Hilbert series to $\mathrm{K}_{\mathrm{T}}$-classes [MS05, chapter 8.2], we can compute

$$
\left.S_{w}\right|_{v}=h_{X_{w} \mid v} \prod_{\beta<0}\left(1-e^{v \cdot \beta}\right)=h_{X_{w o}^{v}} \prod_{\beta<0, v \cdot \beta<0}\left(1-e^{v \cdot \beta}\right)
$$

where the products account for the weights on the T-spaces $N_{+} v B_{+} / B_{+}, v N_{-} B_{+} / B_{+}$respectively.

Corollary 3. [Gr02, Wi06] Let $\mathrm{Q}$ be a reduced word for $v$, and $\Delta(\mathrm{Q}, w)$ the subword complex. Let $\Delta(\mathrm{Q}, w)^{\circ}$ be the set of interior faces, i.e. those $\mathrm{F}$ such that the Demazure product of $\mathrm{Q} \backslash \mathrm{F}$ is exactly $w($ not $>w)$. Then

$$
S_{w \mid v}=\sum_{\mathrm{F} \in \Delta(\mathrm{Q}, w)^{\circ}}(-1)^{|\mathrm{Q} \backslash \mathrm{F}|}\left(\prod_{i=1}^{\ell(v)}\left(\widehat{1-e^{-\alpha_{i}}}\right)^{[\mathrm{i} \in \mathrm{F}]} \mathrm{r}_{\alpha_{i}}\right) \cdot 1
$$

where $\widehat{x}$ is the multiply-by-x operator, and $[i \in F]=0,1$ according to whether the condition fails or is satisfied.

Proof. This is the combination of corollary 1 and the second half of corollary 2 .

This K-theory result implies in turn the corresponding equivariant cohomology result from [AJS94, Appendix D] and [Bi99], which is a sum only over the facets of $\Delta(\mathrm{Q}, w)$, rather than all interior faces.

The following technical lemma gives an inductive way to construct subword complexes. Since it is purely combinatorial, we put it in this section, but its main use will be in the geometry of the next section. 
Lemma 2. Fix $w \leq v \in W$, and let $\mathrm{Q}$ be a reduced word for $v$.

For each $i \leq \ell(v)$, let $v_{i}$ denote the Demazure product of the initial subword $\left(q_{1}, \ldots, q_{i}\right)$, and

$$
C_{i}:=\left\{\left(w^{\prime} \leq v_{i}, S \subseteq\{i+1, \ldots, \ell(v)\}\right): \ell\left(w^{\prime}\right)+|S|=w, w^{\prime} \prod S=w\right\} .
$$

By the length considerations, in each pair $\left(w^{\prime}, S\right) \in C_{i}$ the subword $S$ is automatically a reduced word for $w^{\prime-1} w$. Plainly $C_{\ell(v)}=\{(w, \emptyset)\}$ whereas $C_{0}=\{(1, S \subseteq Q): S$ is a reduced word for $w\}$.

There is a surjection $\mathrm{C}_{i-1} \rightarrow \mathrm{C}_{i}$, taking

$$
\left(w^{\prime}, S\right) \mapsto \begin{cases}\left(w^{\prime}, S\right) & \text { if } i \notin S \\ \left(w^{\prime} r_{\alpha}, S \backslash\{i\}\right) & \text { if } i \in S\end{cases}
$$

Consequently, we can construct $\mathrm{C}_{i-1}$ from $\mathrm{C}_{i}$ as the disjoint union of the fibers of this surjection. Let $\left(w^{\prime}, S\right) \in C_{i}$, and let $\alpha$ be the ith root in the reduced word $\mathrm{Q}$, so $v_{i}=v_{i-1} r_{\alpha}$. Then each fiber has one or two elements:

(1) If $w^{\prime} r_{\alpha}>w^{\prime}$, then $w^{\prime} \leq v_{i-1}$, and $\left(w^{\prime}, S\right) \in C_{i-1}$ as well.

(2) If $w^{\prime} r_{\alpha}<w^{\prime}$ but $w^{\prime} \not \leq v_{i} r_{\alpha}=v_{i-1}$, then $\left(w^{\prime} r_{\alpha},\{i\} \cup S\right) \in C_{i-1}$.

(3) If $w^{\prime} r_{\alpha}<w^{\prime} \leq v_{i} r_{\alpha}=v_{i-1}$, then $\left(w^{\prime}, S\right),\left(w^{\prime} r_{\alpha},\{i\} \cup S\right)$ are both in $C_{i-1}$.

Proof. All the claims made are essentially tautological.

0.4. From \#2 to \#3: Schubert patches degenerate to subword complexes. We come shortly to the principal new theorem, theorem 4 , after an abbreviated history of related results.

There has been a great deal of work on degenerations of Schubert varieties (rather than patches) to unions of toric varieties, starting with Hodge's degeneration of the Grassmannian (and its Schubert varieties) in its Plücker embedding, limiting to what we today would call the projective Stanley-Reisner scheme of the order complex of the Bruhat order on the Grassmannian (see e.g. [DEP82]).

For more general Schubert varieties in more general embeddings, it has been very fruitful to degenerate to unions not just of projective spaces, but of more complicated toric varieties, e.g. [Ch00, Ca02, KogM05]. (The degeneration in [Ch00] of a flag manifold is to a Stanley-Reisner scheme if the original flag manifold is a "minuscule" flag manifold in its fundamental embedding, but not otherwise.) Unlike the geometric results in this paper, the constructions of these degenerations for general $\mathrm{G}$ have depended on deep algebraic results about Lusztig's or Kashiwara's canonical bases.

Much less seems to be known if one insists on Stanley-Reisner schemes. However one may change the game: rather than degenerating Schubert varieties, one may degenerate matrix Schubert varieties [KnM05, KnMY] or Schubert patches [GR06, KodR03, KrL04, RU\#1, RU\#2]. One difference when working with patches is that the choice of embedding becomes immaterial.

The Schubert patches considered to date in this context all live in various flavors of Grassmannians, i.e. minimal flag manifolds of classical groups. We give now a uniform result producing Stanley-Reisner degenerations of arbitrary Schubert patches in finitedimensional G/B. Or rather, we give a result about Kazhdan-Lusztig varieties, which one may multiply by a vector space using lemma 1 if one prefers to work with Schubert patches. 
Theorem 4. Fix $w \leq v \in W$, and let $\mathrm{Q}$ be a reduced word for $v$.

Then there is a sequence of flat T-equivariant degenerations, starting from the Kazhdan-Lusztig variety $X_{w o}^{v}$, and culminating in $\operatorname{SR}(\Delta(\mathrm{Q}, w))$.

Proof. The proof is of course inductive, and we need first to describe the structure of the intermediate cases, in a setting partway between Kazhdan-Lusztig varieties and StanleyReisner schemes of subword complexes.

Let $C_{i}$ be the set of pairs $\left(w^{\prime}, S\right)$ defined in lemma 2 , and let

$$
X_{i}:=\bigcup_{\left(w^{\prime}, S\right) \in C_{i}}\left(X_{w^{\prime} \circ}^{\nu_{i}} \times\left(\mathbb{A}^{1}\right)^{\{i+1, \ldots, \ell(v)\} \backslash S}\right) \subseteq X_{\circ}^{\nu_{i}} \times\left(\mathbb{A}^{1}\right)^{Q}
$$

where $\left(\mathbb{A}^{1}\right)^{\{i+1, \ldots, \ell(v)\} \backslash S}$ denotes the evident coordinate subspace of $\left(\mathbb{A}^{1}\right)^{\mathrm{Q}}$. Then $X_{\ell(v)}=$ $\left.\mathrm{X}_{w}\right|_{v} \times\{0\}$ and $\mathrm{X}_{0}=\left(\mathrm{B}_{+} / \mathrm{B}_{+}\right) \times \mathrm{SR}(\Delta(\mathrm{Q}, w))$.

We can now give the correct inductive claim: for each $i=1, \ldots, \ell(v)$, there is a flat T-equivariant degeneration of $X_{i}$ to $X_{i-1}$. Specifically, we will show that the degeneration described before theorem 2, when applied to the first factor of $X_{i}$, gives $X_{i-1}$.

We first make a general comment about degenerating unions of closed subschemes (here the components of $\mathrm{C}_{\mathfrak{i}}$ ). Set-theoretically, the limit of a union is the union of the limits, but scheme-theoretically there is usually only an inclusion. (Consider two points colliding in a line, whose scheme-theoretic limit is a fat point, containing the reduced union of the limit point with itself.)

In the case at hand, we can follow an individual component $X_{w^{\prime} \circ}^{v_{i}} \subseteq X_{i}$ using theorem 2], and see that it produces exactly the components listed in lemma2 2 in the corresponding fiber of the $C_{i-1} \rightarrow C_{i}$ surjection. By the above comment, we have shown that $X_{i}$ degenerates to a scheme whose reduction is $X_{i-1}$.

This gives an inequality on Hilbert series, with equality exactly if the degeneration is already reduced. Chaining these inequalities together, we get an inequality relating the Hilbert series of $X_{0}$ and $X_{\ell(v)}$. But by corollary 2, we know these Hilbert series are equal. Hence each intermediate degeneration is indeed of $X_{i}$ to $X_{i-1}$, scheme-theoretically.

Chaining these degenerations $X_{\ell(v)} \leadsto \ldots \sim X_{0}$ together, we have the sequence claimed in the theorem.

Hartshorne's connectedness theorem for Hilbert schemes states that two subschemes of the same projective space with the same K-class can be connected by a series of deformations and degenerations. So one might expect the Graham/Willems formula to imply the above theorem directly. But this theorem is better in two ways: all the degenerations preserve the equivariant $\mathrm{K}$-class, and the theorem uses only degenerations (general to special), not deformations (special to general). So one may use semicontinuity arguments, e.g. the Stanley-Reisner schemes of subword complexes being Cohen-Macaulay (since the complexes are shellable) implies that Schubert patches are Cohen-Macaulay. (In [Kn] this argument was used one degeneration at a time.)

It seems likely (though we didn't pursue it) that one could use the reduced word Q to define good coordinates on the opposite cell $X_{\circ}^{v}$, within which the above degeneration is by a Gröbner basis with squarefree initial terms, and that the reduced Gröbner basis could be inductively constructed using the vertex decomposition of the subword complex. A 
basis for this ideal (though not in these specific coordinates) was already constructed in [LLM98, proposition 9.6.1], using Frobenius splitting and canonical basis techniques.

0.5. Acknowledgements. Primarily I thank Rebecca Goldin, with whom we discovered the Graham/Willems formula sometime between [Gr02] and [Wi06]. This work would have been quite impossible without Ezra Miller, who taught me so much about simplicial complexes. Thanks also to Bill Graham for sending me his preprint [Gr02] and the reference to [AJS94].

\section{REFERENCES}

[AK] V. Alexeev, A. Knutson, Complete moduli spaces of branchvarieties, preprint 2006. math. AG/0602626 [AJS94] H. H. Andersen, J. C. Jantzen, W. Soergel, Representations of quantum groups at a pth root of unity and of semisimple groups in characteristic p: independence of p. Astérisque No. 220 (1994).

[BP79] L. J. Billera and J. S. Provan, A decomposition property for simplicial complexes and its relation to diameters and shellings, Second International Conference on Combinatorial Mathematics (New York, 1978), New York Acad. Sci., New York, 1979, pp. 82-85.

[Bi99] S. Billey, Kostant polynomials and the cohomology ring for G/B, Duke Math. J. 96 (1999), no. 1, 205-224.

[Ca02] P. Caldero, Toric degenerations of Schubert varieties, Transform. Groups 7 (2002), no. 1, 5160. math.RT/0012165

[Ch00] R. Chirivì, LS algebras and application to Schubert varieties, Transform. Groups 5 (2000), no. 3, 245-264.

[De74] M. Demazure, Désingularisation des variétés de Schubert généralisées. Ann. Sci. École Norm. Sup. (4) 7 (1974), 53-88.

[GR06] S. R. Ghorpade and K. N. Raghavan, Hilbert functions of points on Schubert varieties in the symplectic Grassmannian, Trans. Amer. Math. Soc. 358 (2006), no. 12, 5401-5423.math. RT/0409338

[Gr02] W. Graham, Equivariant K-theory and Schubert varieties, preprint 2002.

[DEP82] C. De Concini, D. Eisenbud, C. Procesi, Hodge algebras. With a French summary. Astérisque, 91. Société Mathématique de France, Paris, 1982. 87 pp.

[Ja87] J. C. Jantzen, Representations of algebraic groups. Pure and Applied Mathematics, 131. Academic Press, Inc., Boston, MA, 1987.

[KaL79] D. Kazhdan and G. Lusztig, Representations of Coxeter Groups and Hecke Algebras, Invent. Math. 53 (1979), 165-184.

[Kn] A. Knutson, Automatically reduced degenerations of automatically normal varieties, preprint 2007. arXiv:0709.3999

[KnM05] _ E. Miller, Gröbner geometry of Schubert polynomials, Annals of Mathematics 161 (May 2005), 1245-1318. math. AG/0110058

[KnM04] _ , Subword complexes in Coxeter groups, Advances in Mathematics 184 (2004), no. 1,161-176.math.CO/0309259

[KnMY] , A. Yong, Gröbner geometry of vertex decompositions and of flagged tableaux, to appear in J. Reine Angew. Math.math.AG/0502144

[KodR03] V. Kodiyalam, K. Raghavan, Hilbert functions of points on Schubert varieties in the Grassmannian, J. Algebra 270 (2003), 28-54.math. AG/0206121

[KogM05] M. Kogan, E. Miller, Toric degeneration of Schubert varieties and Gel'fand-Cetlin polytopes, Advances in Mathematics 193 (2005), no. 1,1-17.math. AG/0303208

[KosKu90] B. Kostant, S. Kumar, T-equivariant K-theory of generalized flag varieties. J. Differential Geom. 32 (1990), no. 2, 549-603.

[KrL04] V. Kreiman, V. Lakshmibai, Multiplicities of Singular Points in Schubert Varieties of Grassmannians, Algebra, Arithmetic and Geometry with Applications (West Lafayette, IN, 2000), pp. 553-563, Springer, Berlin, 2004.math. AG/0108071

[LLM98] V. Lakshmibai, P. Littelmann, and P. Magyar, Standard monomial theory and applications. In Representation theories and algebraic geometry (Montreal, PQ, 1997), volume 514 of NATO Adv. Sci. Inst. Ser. C Math. Phys. Sci., pages 319-364. Kluwer Acad. Publ., Dordrecht, 1998. 
[MS05] E. Miller, B. Sturmfels, Combinatorial Commutative Algebra, Graduate Texts in Mathematics, 227. Springer-Verlag, New York, 2005.

[RU\#1] K. N. Raghavan, S. Upadhyay, Hilbert functions of points on Schubert varieties in orthogonal Grassmannians. arXiv:0704.0542

[RU\#2] K. N. Raghavan, S. Upadhyay, Initial ideals of tangent cones to Schubert varieties in orthogonal Grassmannians. arXiv:0710.2950

[Wi06] M. Willems, K-théorie équivariante des tours de Bott. Application á la structure multiplicative de la K-théorie équivariante des variétés de drapeaux. Duke Math. J. 132 (2006), no. 2, 271-309. math.AG/0412152

E-mail address: allenk@math.ucsd.edu 